\title{
Desenvolvimento e Validação de uma Escala de Atitude em Relação à Ciência na Psicologia
}

\author{
Bheatrix Bienemann ${ }^{1}$ \\ Pontifícia Universidade Católica, Rio de Janeiro-RJ, Brasil \\ Bruno Figueiredo Damásio ${ }^{1}$ \\ Universidade Federal do Rio de Janeiro, Rio de Janeiro-RJ, Brasil
}

\section{RESUMO}

A história da Psicologia é marcada por uma crise de cientificidade. Em muitos domínios da área, há indiferença e até antipatia relacionada à prática científica. O presente estudo apresenta o desenvolvimento e as propriedades psicométricas de uma Escala de Atitude em Relação à Ciência na Psicologia (EARC-P). A EARC-P avalia como os estudantes e profissionais da Psicologia pensam, como se sentem e buscam se comportar em relação à ciência na Psicologia. Após procedimentos de refinamento, utilizando técnicas de análise fatorial exploratória e confirmatória, a escala final ficou composta por 41 itens divididos em uma estrutura de dois fatores: Iniciativa Pessoal e Crenças e Afetos, com adequados índices de ajuste. Foi demonstrado que os níveis de atitude em relação à ciência se relacionam com necessidade de desenvolvimento pessoal e necessidade de conhecimento, bem como com o fator de personalidade conscienciosidade. Evidências iniciais sugerem adequação da EARC-P para uso no contexto brasileiro.

Palavras-chave: atitude em relação à ciência; psicologia científica; psicometria; evidência de validade.

\section{ABSTRACT - Development and Validation of an Attitude Scale in Relation to Science in Psychology}

The history of psychology is marked by a crisis of scientification. In many domains within the field there is indifference and even antipathy related to scientific practice. The present study presents the development and psychometric properties of an Attitude Scale in Relation to Science in Psychology (ASRS-P). The ASRS-P assesses how students and professionals in psychology think, feel and seek to behave in relation to science in psychology. After refinement procedures using exploratory and confirmatory factor analysis techniques, the final scale was composed of 41 items divided into a two-factor structure: Personal Initiative, and Beliefs and Affects, with adequate adjustment indexes. It has been shown that attitudes in relation to science relate to a need for personal development, a need for knowledge, as well as the personality factor Conscientiousness. Initial evidence suggests adequacy of the ASRS-P for use in the Brazilian context.

Keywords: attitude towards science; scientific psychology; psychometry; validity evidence.

RESUMEN - Desarrollo y Validación de una Escala de Actitud en relación a la Ciencia en Psicología

La historia de la Psicología es marcada por una crisis de cientificidad. En muchos dominios del área existe indiferencia y hasta antipatía relacionada con la práctica científica. El presente estudio describe el desarrollo y las propiedades psicométricas de una Escala de Actitud con relación a la Ciencia en Psicología (EARC-P), cuyo objetivo es evaluar cómo los estudiantes y profesionales de psicología piensan, sienten y actúan con relación a la ciencia en Psicología. Después de procedimientos de refinamiento, utilizando técnicas de análisis factorial exploratorio y confirmatorio, la escala final quedó compuesta por 41 ítems divididos en una estructura de dos factores: Iniciativa Personal (IP) y Creencias y Afectos (CA) con adecuados índices de ajuste. Los niveles de actitud en relación a la ciencia, se relacionan con la necesidad de desarrollo personal y necesidad de conocimiento, así como con el factor de personalidad escrupulosidad (conscientiousness). Evidencias iniciales sugieren la EARC-P como una medida adecuada para ser utilizada en el contexto brasileño. Palabras clave: actitud en relación a la ciencia; psicología científica; psicometría; evidencias de validez.

A Psicologia moderna tem em sua história uma longa e persistente crise relacionada à sua cientificidade (Castañon, 2009a). Essa crise advém de uma série de problemas postulados ao longo do tempo. Alguns desses problemas, dizem respeito a alegações sobre a natureza ontológica da Psicologia ser supostamente incompatível com a prática científica, e boa parte deles foram derivados dos vetos estabelecidos por Immanuel Kant na Crítica da
Razão Pura (1781/2001) ou no prefácio dos Princípios Metafísicos da Ciência da Natureza (1786/1990). Um desses vetos, por exemplo, refere-se à impossibilidade de quantificação dos fenômenos psicológicos, uma vez que Kant nega que a matemática possa ser aplicável a esses fenômenos internos e às leis que os regem. Dessa forma, a Psicologia poderia ser apenas um conhecimento descritivo e não científico (Kant, 1786/1990). 
O método científico nas ciências humanas, entretanto, é análogo ao método das ciências naturais, que, de acordo com Popper (1992), consiste basicamente na tentativa de solução de problemas sujeita ao controle rigoroso da crítica. Dessa forma, não há nada inerente às ciências sociais ou à própria Psicologia que as façam impermeáveis ao método científico. Tão verdadeiro é esse fato que a própria história da Psicologia, bem como o seu momento presente, está repleta de ilustrações de tentativas bem-sucedidas de embasamento empírico e científico. Além disso, diferentes correntes da Psicologia conseguiram encontrar soluções para cada um dos vetos kantianos ao longo da história, demonstrando, definitivamente, que é possível para a Psicologia superar os supostos problemas relativos à sua cientificidade (Castañon, 2009a).

Outros problemas que acentuam a crise de cientificidade da Psicologia derivam da forte influência na área por parte de correntes filosóficas que adotam uma visão epistemológica pós-modernista (Castañon, 2009a), cujos preceitos são incompatíveis e contrários à própria ciência moderna (Barreto, 2012) e que acabam funcionando como uma espécie de "anticiência" (Sagan, 2006/1995). Esse movimento rejeita o projeto de modernidade advindo do iluminismo - o que acaba por se estender a todos os pressupostos que fundamentam as atividades científicas (Castañon, 2009b).

Apesar do inestimável progresso científico e tecnológico avançando cada vez mais rápido sobre o mundo, o mesmo parece não acontecer dentro da área da Psicologia. Alguns profissionais tendem a valorizar mais a experiência clínica e subjetiva, em detrimento de evidências obtidas por pesquisas científicas, além de nutrirem indiferença e até antipatia relacionada ao método científico em muitos domínios da área (Lilienfeld, 2010). Além disso, há estudos demonstrando bastante ceticismo em relação à Psicologia e sua cientificidade por parte da população em geral (Lilienfeld, 2012).

Considerando esse panorama, assume-se indispensável uma prática psicológica que seja baseada em evidências que atestem a sua eficácia. Evidências devem ser buscadas tanto para se sustentar decisões clínicas relacionadas a diagnósticos, bem como para plano de tratamento, intervenções e resultados (Cruz \& Pimenta, 2005). Nesse sentido, são inegáveis os diversos benefícios proporcionados pela atividade científica e pelo consequente desenvolvimento tecnológico à humanidade, ao longo da história.

Entretanto, a necessidade de uma Psicologia que tenha o seu funcionamento asseguradamente testado preconiza uma Psicologia baseada no método científico. Uma vez que o estado atual da ciência na área é, frequentemente, visto como desanimador (Lilienfeld, 2010), é importante compreender como a ciência está sendo inserida na área pelos seus profissionais e estudiosos, bem como o quanto essas pessoas estão dispostas a construírem uma psicologia científica em suas práticas.

\section{Por que medir atitude?}

Atitudes são compostas por três componentes principais, sendo eles o componente cognitivo, afetivo e comportamental (Rodrigues, Assmar \& Jabonski 2001). Ou seja, elas dizem respeito à forma como as pessoas pensam, sentem e se comportam frente a alguém ou a determinado objeto. Ainda que não haja consenso na literatura sobre se as atitudes podem ser tidas como preditores confiáveis do comportamento das pessoas (Fishbein \& Ajzen, 2011; Rodrigues et al., 2001), elas revelam, pelo menos, a maneira como as pessoas gostariam de se comportar frente a algum objeto, e também influenciam, indiretamente, o comportamento por meio da percepção sobre o próprio comportamento e criação de metas (Kruglanski et al., 2015).

Portanto, mensurar atitudes de estudantes e psicólogos diante da ciência na área é entender o que essas pessoas pensam sobre a Psicologia ser uma ciência, como elas se sentem em relação a isso e as suas disposições pessoais para se engajar na construção da Psicologia enquanto ciência. Estudar atitude em relação à ciência na Psicologia (ARCP) é, desse modo, um aprofundamento nas diferentes opiniões sobre o tema. Uma vez que esse mapeamento esteja feito, torna-se muito mais fácil entender em que consiste a resistência à Psicologia científi$\mathrm{ca}$, bem como as maneiras efetivas de se começar a pensar em soluções para lidar com essa resistência.

A mensuração da ARCP serviria como base para a investigação do panorama em que se encontra a ciência dentro do campo da Psicologia e para que, a partir dessa constatação, medidas de valorização da ciência na Psicologia possam começar a ser pensadas e tomadas. Durante o período de desenvolvimento deste estudo e revisão da literatura, não foram encontrados estudos, nacionais ou internacionais, que buscassem avaliar ARCP por meio de uma escala construída para tal. Sendo assim, o objetivo do estudo foi o de construir e apresentar evidências iniciais de validade de uma Escala de Atitude em Relação à Ciência na Psicologia (EARC-P), por meio de análises fatoriais e relações com medidas externas.

No que diz respeito à validade externa do instrumento, foram estabelecidas algumas hipóteses. Esperase que a EARC-P tenha relação com o fator de personalidade Conscienciosidade, uma vez que esse fator agrupa traços relacionados a rigor, boa organização, diligência, responsabilidade, além de se correlacionar com desempenho acadêmico (Sorić, Penezić, \& Burić, 2017). Também se espera que a EARC-P se relacione com Necessidade de Conhecimento e Desenvolvimento Pessoal, facetas do Sources of Meaning and Meaning in Life Questionnaire que enfatizam a busca por desenvolvimento pessoal e de conhecimento ao longo do ciclo vital (Schnell, 2009). Sendo assim, espera-se que uma pessoa com alto nível de engajamento e valorização à ciência na sua área tenha também uma grande predisposição a adquirir conhecimento, o que a permitirá tanto construir 
modelos teóricos quanto derivar hipóteses testáveis deles (Popper, 1992). Além disso, devido ao caráter de atualização constante e autocorreção de erros da ciência (Sagan, 2006/1995), espera-se que o indivíduo engajado na atividade científica também tenha um maior empenho em seu desenvolvimento pessoal, como reflexo da necessidade de se estar em uma constante atualização e reformulação de suas próprias hipóteses.

\section{Método}

Participou dessa pesquisa uma amostra de 611 pessoas, estudantes $(n=345)$ ou profissionais $(n=265)$ da Psicologia. Do total, 392 eram do sexo feminino
(64,2\%). O perfil etário da amostra variou de 18 aos 75 anos $(M=27,51$ e $D P=9,8)$. Com relação ao perfil educacional, a maioria dos participantes tem até graduação completa $(n=402)$, seguido pelos que possuem até mestrado completo $(n=73)$, doutorado $(n=72)$ e especialização $(n=64)$. Dos participantes ainda cursando a graduação $(n=331), 207$ (33,9\%) estão em universidade pública e 124 (20,3\%), em universidade particular. Em relação à região de moradia dos participantes, 330 (54\%) se encontram na região Sudeste, $149(24,4 \%)$ na região Sul, $94(15,4 \%)$ na região Nordeste, 26 (4,3\%) na região Centro-Oeste e 12 (2\%) participantes se encontram na região Norte do país. A descrição dos participantes pode ser vista na Tabela 1.

Tabela 1

Características Sociodemográficas da Amostra

\begin{tabular}{|c|c|c|c|c|c|c|}
\hline \multirow{3}{*}{ Idade } & \multicolumn{2}{|c|}{ Total } & \multicolumn{2}{|c|}{ Homens } & \multicolumn{2}{|c|}{ Mulheres } \\
\hline & $M$ & $D P$ & $M$ & $D P$ & $M$ & $D P$ \\
\hline & 27,01 & 8,09 & 25,74 & 6,35 & 27,72 & 8,84 \\
\hline Idade em categorias & $N$ & $\%$ & $n$ & $\%$ & $n$ & $\%$ \\
\hline $\begin{array}{l}18-25 \text { anos } \\
26-35 \text { anos } \\
36-55 \text { anos } \\
56-65 \text { anos }\end{array}$ & $\begin{array}{c}343 \\
191 \\
70 \\
7\end{array}$ & $\begin{array}{c}56,1 \\
31,3 \\
11,5 \\
1,1\end{array}$ & $\begin{array}{c}133 \\
69 \\
16 \\
1\end{array}$ & $\begin{array}{c}53,6 \\
31,1 \\
13,8 \\
1,5\end{array}$ & $\begin{array}{c}210 \\
122 \\
54 \\
6\end{array}$ & $\begin{array}{c}53,6 \\
31,1 \\
13,8 \\
1,5\end{array}$ \\
\hline Região do Brasil & $N$ & $\%$ & $n$ & $\%$ & $n$ & $\%$ \\
\hline $\begin{array}{l}\text { Sudeste } \\
\text { Sul } \\
\text { Nordeste } \\
\text { Centro-Oeste } \\
\text { Norte }\end{array}$ & $\begin{array}{c}330 \\
149 \\
94 \\
26 \\
12\end{array}$ & $\begin{array}{c}54 \\
24,4 \\
15,4 \\
4,3 \\
2\end{array}$ & $\begin{array}{c}113 \\
49 \\
39 \\
14 \\
4\end{array}$ & $\begin{array}{c}51,6 \\
22,4 \\
17,8 \\
6,4 \\
1,8\end{array}$ & $\begin{array}{c}217 \\
100 \\
55 \\
12 \\
8\end{array}$ & $\begin{array}{c}55,4 \\
25,5 \\
14 \\
3,1 \\
2\end{array}$ \\
\hline Escolaridade & $N$ & $\%$ & $n$ & $\%$ & $n$ & $\%$ \\
\hline $\begin{array}{l}\text { Graduação } \\
\text { Especialização } \\
\text { Mestrado } \\
\text { Doutorado }\end{array}$ & $\begin{array}{l}402 \\
64 \\
73 \\
72\end{array}$ & $\begin{array}{l}65,8 \\
10,5 \\
11,9 \\
11,8\end{array}$ & $\begin{array}{l}150 \\
18 \\
25 \\
26\end{array}$ & $\begin{array}{c}68,5 \\
8,2 \\
11,4 \\
11,9\end{array}$ & $\begin{array}{c}252 \\
46 \\
48 \\
46\end{array}$ & $\begin{array}{l}64,3 \\
11,7 \\
12,2 \\
11,7\end{array}$ \\
\hline Crença Religiosa & $N$ & $\%$ & $n$ & $\%$ & $n$ & $\%$ \\
\hline $\begin{array}{l}\text { Sim } \\
\text { Não }\end{array}$ & $\begin{array}{l}347 \\
264\end{array}$ & $\begin{array}{l}56,8 \\
43,2\end{array}$ & $\begin{array}{c}83 \\
136\end{array}$ & $\begin{array}{l}37,9 \\
62,1\end{array}$ & $\begin{array}{l}264 \\
128\end{array}$ & $\begin{array}{l}67,3 \\
32,7\end{array}$ \\
\hline Estado Civil & $\mathrm{N}$ & $\%$ & $n$ & $\%$ & $n$ & $\%$ \\
\hline Solteiro & 484 & 69,4 & 175 & 79,9 & 254 & 64,8 \\
\hline Casado & 105 & 15,1 & 15 & 6,8 & 74 & 18,9 \\
\hline União Estável & 64 & 9,2 & 17 & 7,8 & 31 & 7,9 \\
\hline Noivo & 21 & 3 & 8 & 3,7 & 15 & 3,8 \\
\hline Divorciado & 21 & 3 & 2 & 0,9 & 16 & 4,1 \\
\hline Viúvo & 2 & 0,3 & 0 & 0 & 1 & 0,3 \\
\hline União Civil & 0 & 0 & 2 & 0,9 & 1 & 0,3 \\
\hline
\end{tabular}

Nota. $\mathrm{M}=$ média; $\mathrm{DP}=$ desvio padrão

\section{Instrumentos}

Escala de Atitude em Relação à Ciência na Psicologia (EARC-P): Procedimentos de Construção.

Inicialmente, foram elaborados 52 itens teoricamente coerentes e relativos a aspectos emocionais (i.e.,
"Como eu me sinto em relação à ciência aplicada à Psicologia"), cognitivos (i.e., "O que eu penso sobre a ciência na Psicologia") e comportamentais (i.e., "Como eu me comporto em relação à ciência na Psicologia") sobre a aplicação da ciência na Psicologia. Ao final dos procedimentos de criação dos itens, avaliou-se a 
pertinência de todos os itens, em relação à sua estrutura gramatical, semântica e idiomática (Borsa, Damásio, \& Bandeira, 2012). Itens repetitivos, semanticamente semelhantes, ou gramaticalmente complexos, foram eliminados. No fim do procedimento, restaram 48 itens na EARC-P.

Os itens foram avaliados por três juízes externos, experts na área de construção de testes, que avaliaram a validade de conteúdo dos itens. Foi observada a clareza dos itens em relação aos seus aspectos linguísticos, se os itens aparentemente medem o que se propõe a medir e se precisariam ser reestruturados ou excluídos. Após ajustes menores realizados por meio das sugestões desses juízes externos, os itens passaram por um estudo piloto, onde uma amostra da população à qual a escala se destina avaliou o instrumento $(\mathrm{N}=12)$. Nesse processo, nenhum item foi eliminado; apenas ajustes gramaticais, semânticos ou idiomáticos foram, mais uma vez realizados, mantendo, portanto, o total de 48 itens na escala. Após a realização do estudo piloto e das alterações na estrutura do instrumento, ela foi considerada pronta para aplicação e posterior avaliação psicométrica (Borsa et al., 2012). O instrumento é respondido em uma escala tipo Likert de cinco pontos, variando de 1 (discordo totalmente) a 5 (concordo totalmente). Quanto maior a pontuação do indivíduo, mais favorável é a sua atitude em relação à ciência na Psicologia.

\section{Instrumentos Adicionais.}

Questionário Sociodemográfico. Esse questionário teve por objetivo levantar informações sociodemográficas, tais como sexo, idade, grau de instrução, local de residência, tipo de instituição de ensino superior a qual o participante estuda/estudou (pública ou privada), local de formação, etc.

Subescala de Necessidade de Desenvolvimento Pessoal. (SNDP; Schnell \& Becker, 2007; Schnell, 2009; adaptada por Damásio \& Koller, 2013). A SNDP refere-se a uma subescala do Sources of Meaning and Meaning in Life Questionnaire (SoMe) - um instrumento que avalia índices de sentido de vida, crises de sentido e fontes de sentido. A SNDP é composta por seis itens que avaliam, em uma escala de seis pontos $(0=$ discordo totalmente $; 5=$ concordo totalmente) a importância de desenvolvimento pessoal na vida dos sujeitos. No presente estudo, a consistência interna da escala (alfa de Cronbach) foi de $\alpha=0,85$.

Subescala de Necessidade de Conhecimento. (SNC; Schnell \& Becker, 2007; Schnell, 2009; adaptado por Damásio \& Koller, 2013). A SNC refere-se, também, à uma subescala do Souces of Meaning and Meaning in Life Questionnaire (SoMe). A SNC é composta por seis itens que avaliam, em uma escala de seis pontos $(0=$ discordo totalmente; 5 = concordo totalmente) a importância ou necessidade de conhecimento na vida dos sujeitos. No presente estudo, a consistência interna da escala (alfa de Cronbach) foi de $\alpha=0,79$.
Marcadores Reduzidos da Personalidade. (MRP, Hauck-Filho, Machado, Teixeira, \& Bandeira, 2012). A MRP é um instrumento de avaliação da personalidade baseada no modelo dos cinco grandes fatores. É composta por 25 itens, respondidos em escala Likert de cinco pontos, variando de 1 (discordo totalmente) a 5 (concordo totalmente). A escala é dividida em cinco fatores, sendo eles: extroversão, socialização, consciensiosidade, neuroticismo e abertura à experiência, sendo que cada construto é mensurado por cinco itens. No estudo de validação original (Hauck-Filho et al., 2012), os autores apresentaram índices de consistência interna satisfatórios, variando de 0,61 (abertura à experiência) até 0,83 (extroversão). No presente estudo, a consistência interna da escala (alfa de Cronbach) foi de $\alpha=0,83$ para Extroversão, $\alpha=0,77$ para Socialização, $\alpha=0,75$ para Conscienciosidade, $\alpha=0,74$ para Neuroticismo e $\alpha=0,61$ para Abertura à Experiência.

\section{Procedimentos de Análise de Dados}

Inicialmente, a amostra foi dividida, de maneira aleatória, em aproximadamente duas partes iguais. Com a primeira parte da amostra $(\mathrm{n}=305)$, foi avaliado, por meio do software Factor v.9.2 (Lorenzo-Seva \& Ferrando, 2006), o melhor número de fatores a ser retido para a EARC-P. Nesse processo, foram utilizados dois critérios distintos, sendo: 1) análise paralela (Horn, 1965) com permutação aleatória dos dados (Timmerman \& Lorenzo-Seva, 2011) e 2) método Hull (Lorenzo-Seva, Timmerman, \& Kiers, 2011). Com base nos resultados encontrados, procedeu-se para avaliar a estrutura fatorial da EARC-P por meio de uma análise fatorial exploratória (Exploratory Factor Analysis, EFA), utilizando o método de estimação Weighted Least Squares Mean-and-variance Adjusted (WLSMV; Muthén \& Muthén, 2012), adequado para variáveis ordinais.

Com base nos resultados da EFA, procedimentos de refinamento da escala foram implementados, com vistas a tornar a escala mais parcimoniosa. Assim, optou-se por excluir os itens que apresentassem cargas fatoriais baixas (abaixo de 0,40 ) ou que tivessem carga cruzada acima de $0,30 \mathrm{em}$ mais de um fator.

Após o refinamento, buscou-se avaliar a adequação da nova estrutura da EARC-P. Para isso, foram realizadas análises fatoriais confirmatórias (AFC) com a segunda parte da amostra $(n=306)$, utilizando o mesmo método de estimação da etapa anterior (WLSMV). Os índices de ajuste utilizados foram o Root Mean Square error of Approximation (RMSEA); Comparative Fit Index (CFI), Tucker-Lewis Index (TLI). De acordo com os critérios utilizados (Brown, 2006), valores de RMSEA ( $90 \%$ IC) não devem ser superiores a 0,10 , e valores de CFI e TLI devem ser maiores que 0,90 , preferencialmente acima de 0,95 . Índices de fidedignidade da escala foram avaliados por meio da fidedignidade composta. 


\section{Procedimentos Éticos e de Coleta de Dados}

A coleta de dados foi realizada virtualmente. $\mathrm{O}$ link para participação na pesquisa foi divulgado em redes sociais e por listas de e-mails. O estudo teve aprovação mediante comitê de ética referente à sua localidade. O questionário da pesquisa só tinha início conforme concordância e aceitação do Termo de Consentimento Livre e Esclarecido (TCLE), presente logo na primeira página da pesquisa. A privacidade dos participantes, bem como o sigilo e confidencialidade dos dados foram garantidas. A coleta de dados só aconteceu mediante a concordância e consentimento dos participantes.

\section{Resultados}

\section{Análise Fatorial Exploratória (EFA)}

Inicialmente, buscou-se investigar a dimensionalidade da EARC-P utilizando, enquanto técnicas de retenção fatorial, a análise paralela (Timmerman \& Lorenzo-Seva,
2011) e o método Hull (Lorenzo-Seva, Timmerman, \& Kiers, 2011). As diferentes técnicas apontaram para dois e um fator, respectivamente, como sendo os mais representativos dos dados. Considerando esses resultados, foram executadas duas EFAs, com o objetivo de investigar a estrutura teórica da EARC-P composta por um e dois fatores (Ver Tabela 2).

Conforme pode ser visto na Tabela 2, na estrutura unifatorial, todos os itens carregam adequadamente. No que se refere à estrutura de dois fatores, é possível observar que os itens 1 e 2 não carregaram adequadamente em nenhum fator, enquanto os itens $6,15,33$, 37 e 41 apresentaram carga cruzada, com carga fatorial acima de 0,30 nos dois fatores. Ainda, os índices de ajuste do modelo de dois fatores apresentam-se mais adequados do que quando comparado com o modelo unifatorial [Unifatorial, $\chi^{2}=3345,922, \mathrm{gl}=1080$; RMSEA $(90 \%$ IC $)=0,08(0,08-0,09)$; CFI $=0,94 ;$ TLI $=0,94$; Dois fatores: $\chi^{2}=2537,857, \mathrm{gl}=1033$; RMSEA $(90 \%$ IC) $=0,07$ $(0,07-0,07) ; \mathrm{CFI}=0,96 ; \mathrm{TLI}=0,96]$.

Tabela 2

Estruturas Fatoriais da EARC-P (Uni e Bidimensional)

\begin{tabular}{|c|c|c|c|c|c|c|c|}
\hline \multicolumn{8}{|c|}{ Estrutura de dois fatores } \\
\hline Itens & $\begin{array}{c}\text { Estrutura } \\
\text { unifatorial }\end{array}$ & Fator 1 & Fator 2 & Itens & $\begin{array}{c}\text { Estrutura } \\
\text { unifatorial }\end{array}$ & Fator 1 & Fator 2 \\
\hline Item 1 & $0,49^{*}$ & 0,26 & 0,27 & Item 25 & $0,86^{*}$ & $0,68^{*}$ & 0,23 \\
\hline Item 2 & $0,40^{*}$ & 0,23 & 0,21 & Item 26 & $0,79^{*}$ & 0,13 & $0,74^{*}$ \\
\hline Item 3 & $0,75^{*}$ & 0,16 & $0,66^{*}$ & Item 27 & $0,91^{*}$ & $0,69^{*}$ & 0,27 \\
\hline Item 4 & $0,84^{*}$ & $0,85^{*}$ & 0,00 & Item 28 & $0,87^{*}$ & $0,97^{*}$ & $-0,10$ \\
\hline Item 5 & $0,83^{*}$ & $0,81^{*}$ & 0,05 & Item 29 & $-0,75^{*}$ & $-0,82^{*}$ & 0,07 \\
\hline Item 6 & $-0,79^{*}$ & $-0,40$ & $-0,46$ & Item 30 & $-0,73^{*}$ & $-0,90^{*}$ & 0,19 \\
\hline Item 7 & $-0,65^{*}$ & $-0,77^{*}$ & 0,12 & Item 31 & $-0,73^{*}$ & $-0,71^{*}$ & $-0,03$ \\
\hline Item 8 & $-0,81^{*}$ & $-0,70^{*}$ & $-0,14$ & Item 32 & $0,75^{*}$ & $0,61^{*}$ & 0,19 \\
\hline Item 9 & $-0,50^{*}$ & $-0,57^{*}$ & 0,06 & Item 33 & $-0,74^{*}$ & $-0,48$ & $-0,32$ \\
\hline Item 10 & $-0,72^{*}$ & $-0,65^{*}$ & $-0,11$ & Item 34 & $0,95^{*}$ & $0,94^{*}$ & 0,02 \\
\hline Item 11 & $0,74^{*}$ & $0,73^{*}$ & 0,04 & Item 35 & $0,94^{*}$ & $0,97^{*}$ & $-0,02$ \\
\hline Item 12 & $0,80^{*}$ & 0,16 & $0,72^{*}$ & Item 36 & $0,85^{*}$ & 0,20 & $0,73^{*}$ \\
\hline Item 13 & $0,82^{*}$ & 0,04 & $0,85^{*}$ & Item 37 & $0,88^{*}$ & 0,38 & 0,58 \\
\hline Item 14 & $0,87^{*}$ & $0,80^{*}$ & 0,09 & Item 38 & $-0,68^{*}$ & $-0,73^{*}$ & 0,03 \\
\hline Item 15 & $-0,77^{*}$ & $-0,39$ & $-0,45$ & Item 39 & $-0,63^{*}$ & $-0,69^{*}$ & 0,05 \\
\hline Item 16 & $0,81^{*}$ & 0,06 & $0,81^{*}$ & Item 40 & $-0,78^{*}$ & $-0,75^{*}$ & $-0,06$ \\
\hline Item 17 & $0,83^{*}$ & 0,03 & $0,87^{*}$ & Item 41 & $0,76^{*}$ & 0,46 & 0,35 \\
\hline Item 18 & $0,68^{*}$ & $-0,22$ & $0,98^{*}$ & Item 42 & $0,78^{*}$ & $0,77^{*}$ & 0,03 \\
\hline Item 19 & $0,74^{*}$ & $-0,00$ & $0,82^{*}$ & Item 43 & $-0,69^{*}$ & $-0,67^{*}$ & $-0,05$ \\
\hline Item 20 & $0,65^{*}$ & 0,08 & $0,65^{*}$ & Item 44 & $0,70^{*}$ & $0,81^{*}$ & $-0,11$ \\
\hline Item 21 & $0,67^{*}$ & 0,27 & $0,48^{*}$ & Item 45 & $0,72^{*}$ & $0,61^{*}$ & 0,15 \\
\hline Item 22 & $0,67^{*}$ & $0,60^{*}$ & 0,11 & Item 46 & $0,87^{*}$ & $0,91^{*}$ & $-0,04$ \\
\hline Item 23 & $0,81^{*}$ & $0,69^{*}$ & 0,16 & Item 47 & $-0,57^{*}$ & $-0,48^{*}$ & $-0,12$ \\
\hline Item 24 & $0,85^{*}$ & $0,67^{*}$ & 0,23 & Item 48 & $-0,76^{*}$ & $-0,53^{*}$ & $-0,28$ \\
\hline
\end{tabular}

Nota. Resultados de duas análises fatoriais distintas, sendo uma unidimensional e outra bidimensional; ${ }^{*}$ fator o qual o item carregou 
Ao avaliar teoricamente os dois fatores, percebe-se que o primeiro fator se refere a cognições e afetos que o indivíduo tenha em relação à ciência na Psicologia, sem ter relação com a pessoa propriamente dita, sendo denominado "Crenças e afetos" (CA); enquanto o segundo fator se refere a comportamentos e disposições pessoais relacionados à ciência na Psicologia, sendo denominado "Iniciativa Pessoal" (IP). Considerando a interpretabilidade e adequação teórica da estrutura de dois fatores, bem como seus adequados índices de ajuste, optou-se por acatar essa estrutura em detrimento da unifatorial. Sendo assim, os processos de refinamento da EARC-P foram implementados de acordo com os resultados da estrutura de dois fatores.

Portanto, conforme mencionado anteriormente, com vistas a tornar a escala mais parcimoniosa, durante o processo de refinamento foram excluídos os itens que apresentaram baixa carga fatorial $(<0,40)$ ou carga cruza$\mathrm{da}$ (carga fatorial $>$ de $0,30 \mathrm{em}$ mais de um fator). Sendo assim, foram excluídos os itens 1, 2, 6, 15, 33, 37 e 41 (Ver Tabela 2), de modo que a EARC-P ficou composta por 41 itens.

\section{Análise Fatorial Confirmatória (AFC)}

Com o objetivo de investigar a adequação da escala refinada, foram executadas, com a segunda parte da amostra, duas análises fatoriais confirmatórias (AFC). A primeira AFC foi implementada em uma estrutura unifatorial; a segunda AFC foi implementada numa estrutura de dois fatores, sem a presença de cargas cruzadas. O critério de composição dos itens em cada um dos fatores da EARC-P deu-se por base nas cargas fatoriais dos itens apresentadas na tabela 2 (itens com carga acima de 0,40 foram considerados como "pertencentes" a determinado fator).

Tabela 3

Índices de Ajuste Confirmatórios da EARC-P em suas Versões de Um e de Dois Fatores

\begin{tabular}{lccccc}
\hline \multicolumn{1}{c}{ Modelos } & $\chi^{2}$ & gl & RMSEA & CFI & TLI \\
\hline Unifatorial & $2466.623^{*}$ & 779 & $0,08(0,08-0,09)$ & 0,94 & 0,94 \\
Dois fatores & $1920.221^{*}$ & 778 & $0,07(0,06-0,07)$ & 0,96 & 0,96 \\
\hline
\end{tabular}

Nota. *valor estatisticamente significativo; $\chi^{2}=$ qui-quadrado; gl=graus de liberdade

Conforme pode ser visto na Tabela 3, a estrutura de dois fatores refinada apresentou índices de ajuste adequados e superiores aos do modelo unifatorial, o que atesta, novamente, que a EARC-P é melhor concebida como um instrumento bidimensional. A confiabilidade dos fatores, medido por meio da fidedignidade composta (FC), apresentou, também, resultados adequados: Fator IP, FC $=0,96$ Fator CA, FC=0,98.

\section{Relação entre a EARC-P e Variáveis Psicológicas}

Foram utilizadas correlações bivariadas de Pearson para verificar a relação entre os escores da EARC-P com variáveis psicológicas, mensuradas por meio da subescala de Desenvolvimento Pessoal (SDP), Subescala de Necessidade de Conhecimento (SNC) e dos Marcadores Reduzidos da Personalidade (MRP). O fator IP da EARC-P apresentou correlação positiva moderada estatisticamente significativa com a SDP, enquanto o fator CA apresentou correlação positiva estatisticamente significativa, porém fraca. Foram encontradas correlações positivas fracas entre a SNC e a EARC-P nos dois fatores (Ver Tabela 4).

No que diz respeito à MRP, o fator Extroversão não apresentou nenhuma correlação estatisticamente significativa com nenhum fator da EARC-P. O fator Socialização apresentou correlação positiva fraca com o fator IP e não apresentou correlação estatisticamente significativa com o fator CA. Conscienciosidade se correlacionou positiva e moderadamente com o fator IP e apresentou correlação fraca com o fator CA. Neuroticismo não apresentou correlações estatisticamente significativas com nenhum fator, enquanto Abertura à Experiência apresentou correlação fraca apenas com o fator IP (Ver Tabela 4).

Tabela 4

Correlações entre a EARC-P e Variáveis Psicológicas

\begin{tabular}{lcl}
\hline \multirow{2}{*}{ Escalas } & \multicolumn{2}{c}{ EARC-P } \\
\cline { 2 - 3 } & IP & CA \\
\hline SDP & $0,40^{* *}$ & $0,33^{* *}$ \\
SNC & $0,36^{* *}$ & $0,24^{* *}$ \\
Extroversão & 0,07 & 0,02 \\
Socialização & $0,15^{* *}$ & 0,05 \\
Conscienciosidade & $0,30^{* *}$ & $0,25^{* *}$ \\
Neuroticismo & $-0,05$ & $-0,08$ \\
Abertura à experiência & $0,12^{* *}$ & $-0,04$ \\
\hline
\end{tabular}

Nota. ${ }^{* *} p<0,01 ;{ }^{*} p<0,05$; Valores sem asterisco=correlações não significativas

\section{Discussão}

O objetivo deste estudo foi apresentar os processos de construção e de validação da Escala de Atitude em Relação à Ciência na Psicologia (EARC-P). Embora haja inúmeras definições diferentes sobre atitude, Rodrigues et al. (2001) conseguiram sintetizá-las afirmando que as atitudes são constituídas de três dimensões principais: o 
componente cognitivo, o componente afetivo e o componente comportamental. $\mathrm{O}$ componente cognitivo se refere aos pensamentos e considera as crenças que o indivíduo tem a respeito de determinado objeto ou de alguém. O componente afetivo é aquele que se refere às emoções e afetos do indivíduo e, por último, o componente comportamental é o que se relaciona com a probabilidade ou tendência do indivíduo de se comportar de determinada maneira em relação àquele objeto. Entretanto, um debate muito presente dentro da Psicologia Social, nos tópicos relacionados a esse tema, é o de que os componentes cognitivo e afetivo são geralmente coerentes entre si, mas nem sempre coerentes com o componente comportamental (Rodrigues et al., 2001). Isso ocorre porque as pessoas nem sempre agem de acordo com as suas crenças e seus sentimentos, o que demonstra uma incoerência entre o comportamento e os outros componentes da atitude. Dessa forma, justifica-se teoricamente a estrutura fatorial encontrada na EARC-P, em que os itens relativos ao componente cognitivo e afetivo se agruparam de maneira coerente entre si em um único fator; e o componente comportamental, representado por itens referentes a ações e disposições para ação, agrupou-se em outro fator, coerentemente com a literatura (Kruglanski et al., 2015).

No que diz respeito às relações entre variáveis psicológicas e ARCP, foram encontradas correlações positivas fracas entre a EARC-P e a subescala de Necessidade de Conhecimento nos dois fatores. Em relação à subescala de Desenvolvimento Pessoal, foi encontrada também uma correlação positiva fraca com o fator CA e uma correlação positiva moderada com o fator IP. Isso significa que há uma relação mínima, porém presente nos indivíduos, entre o gostar e ser favorável à prática científica na Psicologia e reconhecer a importância e necessidade de conhecimento e do desenvolvimento pessoal em suas vidas. Além disso, a correlação moderada entre o fator IP e a SDP evidencia mais ainda o caráter pragmático e referente à construção pessoal do fator IP. Como este é um estudo preliminar, também não foram encontrados estudos que relacionassem ARCP com essas variáveis. Entretanto, esses resultados são esperados teoricamente, uma vez que a necessidade de conhecimento se refere ao quanto o sujeito valoriza e busca levantar conhecimentos de diversas áreas ao longo da vida (Schnell, 2009), sendo uma postura bastante coerente com a busca incessante por novas descobertas e o desenvolvimento de novas tecnologias, inerente do campo científico (Sagan, 2006/1995).

Por fim, tratando-se das características de personalidade, foram encontradas correlações fracas entre o fator Conscienciosidade da MRP e os dois fatores da EARC-P, e correlações desprezíveis entre os fatores Socialização, Abertura à Experiência e o fator IP. Apesar das correlações em questão apresentarem pequeno tamanho de efeito, elas mostram um reflexo de algumas constatações encontradas na literatura; tais como que cientistas tendem a possuir consistentes índices de conscienciosidade (Feist, 1998), sendo então, esperado índices maiores de atitude relacionada à ciência entre pessoas possuidoras desta característica. No mais, é importante considerar que a fraca influência das características de personalidade encontrada nos escores da EARC-P também pode ter ocorrido devido ao fato de que foi utilizada uma escala reduzida para avaliação da personalidade, sendo que, por se tratar de um construto complexo, é possível que poucos itens não capturem a totalidade do fenômeno (Carvalho, Nunes, Primi, \& Nunes, 2012).

\section{Considerações Finais}

Este estudo apresenta potencialidades e limitações. Em relação às potencialidades, esse é o primeiro estudo que busca mensurar ARCP e validar uma escala designada a isso. Até o momento, no Brasil, só havia estudos destinados a avaliar ARC de maneira em geral, sem ser voltada especificamente para a área da Psicologia. A EARC-P é uma escala que pode ser utilizada em diferentes amostras de estudantes e profissionais da Psicologia, e que relaciona o tema da atitude em relação à ciência com esta área especificamente. Além disso, o estudo permite a mensuração de ARCP para se verificar quais características contextuais e pessoais podem estar associadas a maior ou menor favorabilidade à prática psicológica científica por parte dos profissionais e estudantes de Psicologia.

Em relação às limitações deste estudo, nota-se que, por ser um estudo pioneiro, algumas explicações dos resultados residiram mais no campo das hipóteses, por ainda não existir literatura sobre determinados temas. A continuidade na investigação sobre a relação entre os níveis de ARCP e variáveis externas e sociodemográficas é necessária para que se compreenda quais fatores estão atuando como moderadores e/ou moduladores nos níveis de ARCP.

\section{Referências}

Barreto, A. (2012). Pós-modernismo em xeque: Alan Sokal e Jean Bricmont em Imposturas Intelectuais. Griot, Revista de Filosofia, 5(1), 154165. Recuperado de http://www2.ufrb.edu.br/griot/component/phocadownload/category/6-vol-05-n-01-junho-2012

Borsa, J. C., Damásio, B. F., \& Bandeira, D. R. (2012). Adaptação e validação de instrumentos psicológicos entre culturas: algumas considerações. Paidéia, 22(53), 423-432. doi: 10.1590/1982-43272253201314 
Brown, T. A. (2006). Confirmatory factor analysis for applied research. New York: The Guilford Press. Recuperado de http://books.google.com/books Castañon, G. A. (2009a). Psicologia como ciência moderna: vetos históricos e status atual. Temas em Psicologia, 17(1), 21-36.

Castañon, G. A. (2009b). Uma guerrilha contra a ciência moderna. Boletim Interfaces da Psicologia da UFRuralRJ, 70. Recuperado de http:// www.ufrrj.br/seminariopsi/2009/boletim2009-1/boletim.pdf

Carvalho, L. F., Nunes, M. F. O., Primi, R., Nunes, C. H. S. S. (2012). Evidências Desfavoráveis para Avaliação da Personalidade com um Instrumento de 10 Itens. Paidéia, 22(51), 63-71. doi:10.1590/S0103-863X2012000100008

Cruz, D. A. L. \& Pimenta, C. A. M. (2005). Prática baseada em evidências, aplicada ao raciocínio diagnóstico. Revista Latino-americana de Enfermagem, 13(3), 415-22. Recuperado de http://dms.ufpel.edu.br/ares/handle/123456789/27

Damásio, B. F., Koller, S. H., \& Schnell, T. (2013). Sources of Meaning and Meaning in Life Questionnaire (SoMe): Psychometric properties and sociodemographic findings in a large Brazilian sample. Acta de Investigación Psicológica, 3(3), 1205-1227. doi: 10.1016/ S2007-4719(13)70961-X

Feist, G. J. (1998). A meta-analysis of personality in scientific and artistic creativity. Personality and social psychology review, 2(4), $290-309$. doi: $0.1207 /$ s15327957pspr0204_5

Fishbein, M., \& Ajzen, I. (2011). Predicting and changing behavior: The reasoned action approach. New York: Taylor \& Francis.

Hauck-Filho, N. H., de Lara Machado, W., Teixeira, M. A. P., \& Bandeira, D. R. (2012). Evidências de Validade de Marcadores Reduzidos para a Avaliação da Personalidade no Modelo dos Cinco Grandes Fatores. Psicologia: Teoria e Pesquisa, 28(4), 417-423. Recuperado de https://revistaptp.unb.br/index.php/ptp/article/view/501

Horn, J. L. (1965). A rationale and technique for estimating the number of factors in factor analysis. Psychometrika, 30(1), 179-185. doi: 10.1007/BF02289447

Kant, I. (1990). Princípios Metafísicos da Ciência da Natureza. Lisboa: Edições 70 (Trabalho original publicado em 1786).

Kant, I. (2001). Crítica da razão pura. Lisboa: Calouste Gulbenkian (Trabalho original publicado em 1781).

Kruglanski, A. W., Jasko, K., Chernikova, M., Milyavsky, M., Babush, M., Baldner, C., \& Pierro, A. (2015). The rocky road from attitudes to behaviors: Charting the goal systemic course of actions. Psychological review, 122(4), 598. doi: 10.1037/a0039541

Lilienfeld, S. O. (2010). Can psychology become a science? Personality and Individual Differences. 49, 281-288. doi: 10.1016/j.paid.2010.01.024

Lilienfeld, S. O. (2012). Public Skepticism of Psychology: Why Many People Perceive the Study of Human Behavior as Unscientific. American Psychologist, 67(2), 111-129. doi: 10.1037/a0023963

Lorenzo-Seva, U., \& Ferrando, P. J. (2006). Factor (9.2) [Computer program]. Recuperado de http://psico.fcep.urv.es/utilitats/factor/

Lorenzo-Seva, U., Timmerman, M. E., \& Kiers, H. A. (2011). The hull method for selecting the number of common factors. Multivariate Behavioral Research, 46(2), 340-364. doi:10.1080/00273171.2011.564527

Muthén, L K, \& Muthén, B. O. (2012). Mplus: Statistical analysis with latent variables. User's guide. Los Angeles: Muthén \& Muthén. Recuperado de https://www.statmodel.com/download/usersguide/MplusUserGuideVer_7.pdf

Popper, K. (1992). The logic of Social-Sciences. Voprosy Filosofii, (10), 65-75. Recuperado de http://www.autodidactproject.org/other/ positivismusstreit/popper_logic_social_sci.pdf

Rodrigues, A., Assmar, E. M. L. \& Jabonski, B. (2001). Psicologia Social. [23 ed.]. Petrópolis: Vozes.

Sagan, C. (2006). O mundo assombrado pelos demônios: a ciência vista como uma vela no escuro. (Eichemberg, R., Trad.). São Paulo: Companhia das letras. (Obra original publicada em 1995).

Schnell, T. (2009). The Sources of Meaning and Meaning in Life Questionnaire (SoMe): Relations to demographics and well-being. The Journal of Positive Psychology, 4(6), 483-499. doi: 10.1080/17439760903271074

Schnell, T., \& Becker, P. (2007). Der Fragebogen zu Lebensbedeutungen und Lebenssinn (LeBe) [The Sources of Meaning and Meaning in Life questionnaires]. Gottingen, Germany: Hogrefe.

Sorić, I., Penezić, Z., \& Burić, I. (2017). The Big Five personality traits, goal orientations, and academic achievement. Learning and Individual Differences, 54, 126-134. doi: 10.1016/j.lindif.2017.01.024

Timmerman, M. E., \& Lorenzo-Seva, U. (2011). Dimensionality assessment of ordered polytomous items with parallel analysis. Psychological Methods, 16, 209-220. doi: 10.1037/a0023353

Anexo A

Escala de Atitude em Relação à Ciência na Psicologia

O questionário a seguir contém uma série de afirmações relacionadas à aplicação da ciência na Psicologia. Gostaríamos que você respondesse sinceramente o quanto você concorda ou não com cada uma destas afirmações. Lembre-se que os dados serão mantidos em sigilo e que não existe resposta certa ou errada, o que importa para nós é a sua opinião.

1 - Discordo totalmente / 2 - Discordo / 3 - Não concordo nem discordo / 4 - Concordo / 5 - Concordo totalmente

Por favor, assinale a opção que mais se enquadra com a sua opinião em relação a cada uma das afirmativas abaixo:

1. Gosto de estudar abordagens científicas na Psicologia.

2. A Psicologia deveria assumir o compromisso de ser mais científica.

3. Defendo o uso de métodos científicos na construção do conhecimento psicológico.

4. Acredito que a ciência não dá conta do estudo da natureza humana.

5. Não vejo necessidade de que a Psicologia seja considerada científica.

6. Crenças pessoais deveriam ser mais importantes do que evidências científicas na Psicologia.

7. A ciência é irrelevante para a Psicologia.

8. O método científico é uma maneira eficaz de se comprovar teorias psicológicas. 
(continuação)

9. Busco me envolver em atividades relacionadas à Psicologia científica.

10. Eu gostaria de ser um(a) cientista na área da Psicologia.

11. O método científico é a maneira mais eficaz para se promover avanços na Psicologia.

12. Eu apreciaria fazer pesquisas científicas na Psicologia.

13. Gosto de ler sobre ciência aplicada à Psicologia.

14. Busco me manter atualizado(a) sobre avanços científicos na Psicologia.

15. Iria a congressos de Psicologia científica por vontade própria.

16. Costumo compartilhar notícias relacionadas à ciência na Psicologia.

17. Dinheiro gasto em pesquisas científicas relacionadas à Psicologia é dinheiro bem investido.

18. Eu me sinto desconfortável quando um profissional da Psicologia se posiciona contrariamente à ciência.

19. A ciência é uma ferramenta eficaz para a compreensão de fenômenos na Psicologia.

20. Acredito que a ciência possa trazer benefícios à Psicologia.

21. Acredito que a pesquisa científica seja fundamental para o desenvolvimento da Psicologia.

22. Eu me sinto animado ao conhecer linhas científicas na Psicologia.

23. Na minha atuação profissional, optaria por utilizar técnicas baseadas em evidências científicas.

24. Eu ficaria mais aliviado(a) se toda a Psicologia fosse baseada em evidências científicas.

25. Acredito que na Psicologia não seja necessário o método científico para se comprovar hipóteses.

26. Não me importo que existam teorias sem evidência científica na minha área.

27. O objeto de estudo da Psicologia não pode ser analisado cientificamente.

28. Eu confio no método científico aplicado à Psicologia.

29. Eu me sinto mais confiante em relação a um estudo da Psicologia que tenha utilizado o método científico.

30. Fico aliviado(a) quando sei que serviços psicológicos são baseados em evidências científicas.

31. Procuro saber mais sobre a Psicologia científica.

32. O método científico prejudica a obtenção de conhecimento na Psicologia.

33. Eu não mudaria minhas opiniões relacionadas à Psicologia caso elas se mostrassem falsas do ponto de vista científico.

34. No geral, a ciência aplicada à Psicologia traz mais prejuízos do que benefícios.

35. Explicações científicas na área da Psicologia são mais válidas do que outros tipos de explicações.

36. Não acho importante entender aspectos psicológicos através da ciência.

37. Sem base científica a prática psicológica seria inútil.

38. Deveria ser obrigatório o aprendizado de Psicologia com base científica na faculdade.

39. O(a) psicólogo(a) deve dar preferência a práticas baseadas em evidências científicas na sua atuação.

40. A ciência aplicada à Psicologia só é útil para pesquisadores.

41. Eu me sinto mal só de pensar em Psicologia com base científica.

\section{Sobre os autores}

Bruno Figueiredo Damásio é Professor do Programa de Pós-Graduação em Psicologia do Instituto de Psicologia da Universidade Federal do Rio de Janeiro. Departamento de Psicometria. Coordenador do Laboratório de Psicometria e Psicologia Positiva (LP3).

Bheatrix Bienemann é Psicóloga formada pela Universidade Federal do Rio de Janeiro. Mestra e doutoranda em Psicologia Clínica pela Pontifícia Universidade Católica do Rio de Janeiro. 